\title{
Radiologic evaluation using computed tomography for compensatory lung growth; a prospective comparison with histological data from a large animal model
}

\section{Keiji Ohata}

Kyoto University Graduate School of Medicine

Toyofumi F. Chen-Yoshikawa

Nagoya University Graduate School of Medicine

Masatsugu Hamaji

Kyoto University Graduate School of Medicine

Takeshi Kubo

Kyoto University Graduate School of Medicine

Tatsuo Nakamura

Kyoto University

Hiroshi Date ( $\nabla$ hdate@kuhp.kyoto-u.ac.jp )

Kyoto University Graduate School of Medicine

\section{Research Article}

Keywords: computed tomography, radiology, compensatory lung growth

Posted Date: May 13th, 2021

DOl: https://doi.org/10.21203/rs.3.rs-498601/v1

License: (c) (i) This work is licensed under a Creative Commons Attribution 4.0 International License.

Read Full License 


\section{Abstract}

Background Non-invasive analysis using computed tomography (CT) data may be a promising candidate to evaluate neo-alveolarization in adult lungs following lung resection. This study evaluates and compares the validity of CT analysis with histologic morphometry for compensatory lung growth in a large animal model.

Methods We calculated the radiologic tissue volume and the radiologic lung weight from CT data taken at 1, 3, and 6 months post-surgery on 15 male beagle dogs that had a right thoractotomy, bilobectomy, or pneumonectomy ( $n=5$ in each group). Results were analyzed using one-way ANOVA and were subsequently compared to histologic findings of tissue samples at 6 months post-surgery using Pearson's correlation.

Results An increase in radiologic tissue volume and radiologic lung weight was identified, which was positively correlated with histologic lung parenchymal amounts (correlation coefficient $=0.955$ and 0.934 , respectively, $p<0.001$ ). Histology of lung specimens at six months post-surgery revealed an increase in the tissue amount in both Bilobectomy and Peumonectomy groups, which was consistent with compensatory lung growth.

Conclusion Radiologic tissue volume and radiologic lung weight reflected compensatory lung growth following lung resection. Radiologic assessment using CT data can be a promising clinical modality to evaluate postoperative lung growth.

\section{Introduction}

Formation of new alveoli (neo-alveolarization) in the adult lungs following lung resection remains a controversial topic. It is generally believed that the process of increasing alveolar numbers is completed until approximately 8 years old in humans, and that this number remains constant following completion ${ }^{1,2}$. However, Bulter et al. have recently reported the occurrence of alveolar regeneration in the residual lung of a 33-year-old female following right pneumonectomy using hyperpolarized helium-3 magnetic resonance imaging ${ }^{3}$. This in turn suggests that neo-alveolarization can occur even in adult lungs, if the appropriate conditions are met. Despite the fact that further detailed studies on human lungs are warranted so as to validate this phenomenon, it is very difficult to perform frequent histologic evaluations with lung biopsies in clinical practice, especially in postoperative states. Therefore, it is essential to develop novel, non-invasive and analytic methodologies.

Radiologic evaluation using computed tomography (CT) scanning is a candidate for the effective evaluation of neo-alveolarization. CT scanning is one of the most frequently performed examinations in current clinical practice that can assess postoperative lung function in an efficient manner. Its highly improving resolution due to recent technological advancements enables qualitative assessment of lung architectures. In fact, this imaging method has been already utilized to assess the severity of emphysema ${ }^{4}$, idiopathic pulmonary fibrosis ${ }^{5}$, and acute respiratory distress syndrome ${ }^{6}$. Hsia et al. 
suggested the effectiveness of "tissue volume" term in large animal studies, and, correspondingly, we proposed the term "radiologic lung weight" in human data analysis as calculated values using CT in compensatory lung growth $7,8,9,10,11$. However, these radiologic measurements are still not universally accepted in regenerative studies. Consequently, the aim of this study is to evaluate the validity of CTderived "radiologic tissue volume" and "radiologic lung weight" prospectively by means of comparison with histologic morphometry in a large animal model.

\section{Results}

\section{Radiologic lung volumetry and measurement of lung tissue amount measurement with radiologic tissue volume and radiologic lung weight}

\section{Lung Volumetry In Radiologic Analysis}

None of the animals included in this study had surgery-related death or comorbidity. The representative images of each group and the time course of lung volume measured from the CT data are shown in Figs. $1 \mathrm{a}$ and $\mathrm{b}$, respectively. Lung volumes at six months post-surgery in the Sham, Bilobectomy, and Pneumonectomy groups were $582.5 \pm 55.3 \mathrm{ml}, 791.8 \pm 102.4 \mathrm{ml}$, and $1100.8 \pm 238.1 \mathrm{ml}$, respectively. Furthermore, the increase rates were $4.7 \pm 7.5 \%, 26.2 \pm 11.3 \%$, and $73.5 \pm 5.2 \%$, respectively. The rates of increase were significantly higher in the Pneumonectomy group than in the other two groups $(p<0.001$, each), and the increase rates in the Bilobectomy group were significantly higher than that in the Sham group $(p=0.004)$.

\section{Lung tissue amount in radiologic analysis}

\section{i. Radiologic tissue volume}

Figures $2 \mathrm{a}$ and $\mathrm{b}$ exhibit the value changes of the fractional tissue volume and the radiologic tissue volume, respectively.

The change rates of fractional tissue volume, reflecting the density of lung tissue, were $98.8 \pm 2.2 \%$ in the Pneumonectomy group, $106.3 \pm 7.0 \%$ in the Bilobectomy group, and $98.2 \pm 7.3 \%$ in the Sham group at six months post-surgery. The fractional tissue volume showed a change rate of $6.9 \%$ which was significantly lower than the $32.5 \%$ change rate in the lung volume ( $p=0.0025$, Fig. $2 a)$.

The values of the radiologic tissue volume at six months post-surgery in the Sham, Bilobectomy, and Pneumonectomy groups were $97.2 \pm 6.1 \mathrm{ml}, 121.7 \pm 11.7 \mathrm{ml}$, and $163.0 \pm 17.4 \mathrm{ml}$, and the respective rates of increase were $2.4 \pm 2.6 \%, 30.7 \pm 7.4 \%$, and $71.5 \pm 7.4 \%$, respectively. The rates of increase were significantly higher in the Pneumonectomy group than in the other two groups ( $p<0.001$, each, Fig. $2 b)$, and the rate of increase in the Bilobectomy group was significantly higher than the one found in the Sham group. These findings are consistent with our results pertaining to the radiologic tissue volume.

\section{ii. Radiologic lung weight}


The values of radiologic lung weight showed the same tendencies with the values of the radiologic tissue volume (Fig. 2c, d). More specifically, radiologic lung weight values at six months after surgery were $102.9 \pm 6.5 \mathrm{~g}, 128.6 \pm 11.9 \mathrm{~g}$, and $172.5 \pm 18.6 \mathrm{~g}$ in the Sham, Bilobectomy, and Pneumonectomy groups, respectively. Furthermore, the rates of increase in the postoperative six months were $2.0 \pm 2.7 \%, 30.3 \pm$ $7.2 \%$, and $71.3 \pm 7.0 \%$, respectively. The rates of increase were significantly higher in the Pneumonectomy group compared to the other two groups $(p<0.001)$, and the rates of increase in the Bilobectomy group was significantly higher than the rate in the Sham group $(p<0.001)$. They showed the same tendencies with radiologic tissue volume.

\section{iii. Correlation between radiologic tissue volume and radiologic lung weight}

There was a strong correlation between radiologic tissue volume and radiologic lung weight (correlation coefficient $=0.929,95 \% \mathrm{Cl} 0.795-0.977, p<0.001$, Fig. 2e).

\section{The histologic evaluations of the residual lungs after lung resections}

\section{The anatomical lung volume of the fixed residual lungs at six months after surgery}

Anatomical lung volume of the fixed left lungs at six months after surgery of the Sham, Bilobectomy, and Pneumonectomy groups were $180.6 \pm 13.1 \mathrm{ml}, 295.4 \pm 36.0 \mathrm{ml}$, and $425.4 \pm 71.6 \mathrm{ml}$, respectively (Fig. 3a). The lungs of the Pneumonectomy group showed greater anatomical volumes than those of the Bilobectomy and Sham groups (Pneumonectomy vs Bilobectomy: $p=0.002$, Pneumonectomy vs Sham: $p<0.001)$, and the Bilobectomy group had greater anatomical volumes compared to the Sham group ( $p=$ $0.006)$.

\section{Histologic Lung Parenchymal Amount}

Representative histologic findings of the residual left lungs of the Sham, Bilobectomy, and Pneumonectomy groups are shown in Fig. 3b. No apparent alveolar hyperinflation was observed in the Bilobectomy and Pneumonectomy groups despite the large increases in anatomical lung volume (Fig. 3b,

c). There was not obvious vascular dilation in the alveolar region of the Bilobectomy and Pneumonectomy groups. The histologic parenchymal amounts of the Sham, Bilobectomy, and Pneumonectomy groups were $44.0 \pm 5.0 \mathrm{ml}, 67.6 \pm 9.3 \mathrm{ml}$, and $96.5 \pm 13.3 \mathrm{ml}$, respectively (Fig. $3 \mathrm{~d}$ ). The Pneumonectomy group exhibited greater histologic parenchymal amount than the Bilobectomy and Sham groups (Pneumonectomy vs Bilobectomy: $p=0.002$, Pneumonectomy vs Sham: $p<0.001$ ), and the Bilobectomy group had larger histologic parenchymal amount compared to the Sham group $(p=0.007)$.

\section{Comparison Between Histologic And Radiologic Results}

Both radiologic tissue volume and radiologic lung weight were significantly positively correlated with histologic parenchymal amounts (correlation coefficient $=0.955$ and 0.934 , respectively, and $p<0.001$ each, Fig. 4).

\section{Discussion}


This study is the first report that assessed the validity of performing CT evaluations regarding qualitative alteration in the residual lungs after lung resection in a large animal model. Our results demonstrate that the residual lungs demonstrate compensatory lung growth in the relatively young canine model after major lung resections. Also, it is shown that CT can provide efficient information that reflects the amount of lung parenchymal amount following major lung resections. The previously reported two calculated values regarding "radiologic tissue volume"7,8,9 and "radiologic lung weight" ${ }^{10}$, were significantly correlated with the histologic parenchymal amount and worked similarly as effective evaluation methods of compensatory lung growth. Our results support the validity of the radiologic evaluation using CT data.

One of the largest issues in the clinical lung growth study is that there is no standard evaluation method to assess lung qualitative changes. Radiologic analysis using CT data may be ideal for compensatory lung growth assessment for several reasons. First, CT scanning is a minimally invasive examination of lung structures even for postoperative patients. Therefore, histologic assessment of tissue samples obtained from invasive lung biopsies can be avoided. Second, CT scanning evaluates the entire lung and compensatory lung growth is considered to provide heterogeneity due to regional lung expansion. CT data cover the entire area of the lungs, and thus have the capacity to evaluate this region in a more precise manner compared to a biopsy. Thirdly, CT analysis is an objective and highly reproducible assessment between examiners, whereas spirometry often provides inaccurate results, especially when it comes to postoperative patients. Furthermore, various CT modalities has been widely used in the field of thoracic surgery such as CT volumetry and three-dimensional CT angiography ${ }^{12,13}$.

Radiologic tissue volume and radiologic lung weight were similarly correlated with the histologic parenchymal amount in compensatory lung growth. The acquired values primarily consist of two factors: lung volume and parenchymal proportion of alveoli. CT number, the value relating radiation absorption in one voxel, is the most important information obtained from CT scanning ${ }^{6}$. The number of air is set as $-1000 \mathrm{HU}$ and the number of water as $0 \mathrm{HU}$, so that the $\mathrm{CT}$ number is expected to correlate with the fraction of parenchymal amount of lung composed of air and soft tissue such as alveoli, bronchioles, and vessels based on the predicted linear relationship between CT number and actual density.

We employed ImageJ software for histologic lung morphometry, and our preliminary study demonstrates a strong correlation with the conventional point counting method (Supplemental material 1). The conventional point counting method is still used to estimate the volume fractions of alveolar septa. In fact, our lung parenchymal proportion measurements of the entire area may be more objective and easyto-perform compared to conventional measurements.

The pneumonectomy model has played a pivotal role in the characterization of the physiological and structural adaptation of the lung to the loss of functional gas-exchange units observed in clinical lung resections and in a number of chronic destructive lung diseases ${ }^{14}$. Hsia et al. reported that right pneumonectomy (removing 55-58\% of total lung) elicited the growth of new acinar tissue in the remaining lung ${ }^{15}$, however, left pneumonectomy (removing 42-45\%) increased the remodeling of the remaining lung structure in canine ${ }^{16}$. In this study, we show that both right pneumonectomy and resection 
of lower and cardiac lobes, which removes only $32 \%$ of total lung, can promote neo-alveolarization. The amount of lung parenchyma increased, and the proportion of parenchyma was higher than the predicted values based on the anatomical lung volume increase ratio. Our data are consistent with a significant increase in alveolar number in canine lungs. This is perhaps due to the different types and ages of the dogs used and due to different surgical procedures performed such as the anterior mediastinal dissection that facilitates midline shift. Our results indicate the possibility that clinical lobectomy could elicit neoalveolarization. Lung resections are a standard treatment for a number of diseases. However, several patients are unable to undergo this procedure due to poor or marginal respiratory function. Consequently, our findings may be applied to clinical practice because of the usage of mammalian young adults that did not grow in height.

There are several limitations in this study. CT scanning was performed in the strictly conditioned situation. Pulmonary infiltrates such as in cases of pneumonia can undermine the accuracy of this modality in clinical practice. Furthermore, we did not perform lung biopsies in during the postoperative period of time so as not to promote any post-procedural complications. Therefore, we could not evaluate potential histologic changes, including molecular events that may have occurred immediately after surgery. After lung resection, the blood flow into residual lung lobes increases. Large vessels are excluded in both radiologic and histologic analyzes, but small vessels in the alveolar region may affect the results.

In conclusion, the histological examinations performed verify that the increase in radiologic tissue volume and radiologic lung weight reflect compensatory lung growth following lung resection. Radiologic assessment using CT data can be a very promising clinical method to evaluate postoperative compensatory of the lung growth.

\section{Methods}

\section{Animals}

Male beagle dogs aged 1 year and 10-11 months and weighing 9-12 kg were used in this study. This study was carried out in compliance with the ARRIVE guidelines (http://www.nc3rs.org.uk/page.asp? id=1357). All animals received care in compliance with the Principles of Laboratory Animal Care (National Society for Medical Research) and the Guide for the Care and Use of Laboratory Animals (National Institutes of Health Publication No. 86 - 23, revised 1996, Bethesda, MD). All surgical procedures were performed by Japanese board-certified thoracic surgeons in accordance with the Guide for the Care and Use of Laboratory Animals published by the National Institutes of Health (NIH Publication No. 85 - 23, revised 1985). The experimental protocol was approved by the Animal Experimental Committee of Kyoto University.

\section{Study Design}

The animals were randomly assigned into one of the following three groups: (i) the Sham group, where only right thoracotomies were performed, (ii) the Bilobectomy group, where only resections of the right lower and cardiac lobes were performed, and (iii) the Pneumonectomy group, where only right 
pneumonectomies were performed ( $n=5$ in each group). The mean ages of the Sham, Bilobectomy, and Pneumonectomy groups at the time of surgery were $22.2 \pm 0.4$ months, $22.6 \pm 0.5$ months, and $22.0 \pm 0$ months, respectively. The mean body weights of each group were $10.4 \pm 0.2 \mathrm{~kg}, 10.5 \pm 1.3 \mathrm{~kg}$, and $10.2 \pm$ $1.0 \mathrm{~kg}$, respectively. There was no difference between three groups in the ages or body weights.

Chest CT scans to all animals preceded surgical procedures in order to establish a baseline. CT scans were also taken at the postoperative time-points of 1,3, and 6 months after surgery. Following 6 months of postoperative scanning, all animals were euthanized, and the lung-heart blocks were procured. Radiologic evaluations and histological findings at the postoperative month six were then compared to assess the validity of radiologic evaluations. (Fig. 5)

\section{CT Scan}

All examinations were performed using a multi-detector CT scanner (Aquilion 64; Canon Medical Systems, Tochigi, Japan) and the images were archived in Digital Imaging and Communications in Medicine format. Axial CT scans were taken with 1-mm thick slices.

Animals were fasted overnight, and they were anesthetized with a subcutaneous administration of 15 $\mathrm{mg} / \mathrm{kg}$ ketamine hydrochloride, $7 \mathrm{mg} / \mathrm{kg}$ xylazine, and $0.5 \mathrm{mg} /$ body atropine sulfate. Subsequently, they were intubated with an $8.5-\mathrm{mm}$ cuffed endotracheal tube and were mechanically ventilated. A 500-mg dose of ampicillin was subcutaneously injected and general anesthesia was maintained with inhalational sevoflurane. CT scans were performed in the supine position, and a pressure-limited respirator was used to maintain the animals' breath at transpulmonary pressure of $20 \mathrm{~cm} \mathrm{H}_{2} \mathrm{O}$ (Evita 4; Dräger, Lubeck, Germany).

\section{Operation Procedure}

After the preoperative CT scan, the right 5th intercostal thoracotomy was performed in the lateral decubitus position. In the Bilobectomy and Pneumonectomy groups, resection of right lower and cardiac lobes and of the right pneumonectomy were performed, respectively; pulmonary arteries and veins were dissected and ligated with 3-0 silk sutures and bronchus with No. 2 silk suture. Anterior mediastinal tissue was dissected to facilitate postoperative midline shift in all groups. The bony thorax was closed with No. 2 polyglactin stiches (Vicryl®, Ethicon, Inc., NJ, USA) and the muscles and skin were closed in layers with 3-0 polyglactin stiches. An additional 500-mg dose of ampicillin was injected subcutaneously at the end of surgery.

\section{Radiologic Evaluation}

CT images at transpulmonary pressure of $20 \mathrm{~cm} \mathrm{H}_{2} \mathrm{O}$ were transferred to the AZE Virtual Place Lexus workstation (Canon Medical Systems, Tochigi, Japan). The workstation automatically executed the segmentation of the left lung and calculated the volume $(\mathrm{ml})$ and the mean CT number (HU) of the extracted volume. The trachea and the next three generations of large conducting airways were excluded 
in the lung segmentation. Then, manual measurement of the CT number of tracheal air and skeletal muscle was performed to determine the estimated air and soft tissue CT number, respectively. The estimated air CT number was calculated by averaging the CT number of three separate regions of the tracheal lumen in each animal. The estimated air-free lung tissue CT number, i.e., estimated soft tissue CT number, was calculated by averaging the CT number of the infraspinatus, supraspinatus, and pectoralis muscles at the level of the carina.

\section{Radiologic tissue volume}

The fractional tissue volume and the radiologic tissue volume were calculated with the average CT numbers of the lung, lung volume, and estimated air density and estimated soft tissue density 7,8,9,17,18. The fractional tissue volume, which represents a tissue density of lung tissue, was determined as follows:

Fractional tissue volume $=\frac{\text { average } C T \text { number of lung }- \text { estimated air number }}{\text { estimated soft tissue number }- \text { estimated air number }}$

In contrast, the CT-derived radiologic tissue volume $(\mathrm{ml})$, which represents the amount of lung tissue, was determined as follows:

Radiologic tissue volume $=$ radiologic lung volume $\times$ fractional tissue volume

\section{Radiologic lung weight}

Radiologic lung weight was calculated with the modified protocol that was described in a previous report $^{10}$, in which, the CT number of air of each scan was used for standardization. The average radiologic lung density $(\mathrm{g} / \mathrm{ml})$, which represents an estimated tissue density of lung tissue, was determined as follows:

Radiologic tissue volume $=$ radiologic lung volume $\times$ fractional tissue volume

\section{Radiologic lung weight}

Radiologic lung weight was calculated with the modified protocol that was described in a previous report ${ }^{10}$, in which, the CT number of air of each scan was used for standardization. The average radiologic lung density $(\mathrm{g} / \mathrm{ml})$, which represents an estimated tissue density of lung tissue, was determined as follows:

Average radiologic lung density $=\frac{\text { mean } C T \text { number }- \text { estimated air number }}{\mid \text { estimated air number } \mid}$

Consequently, the radiologic lung weight $(\mathrm{g})$, which represents the amount of lung tissue, was determined as follows:

Radiologic lung weight = lung volume $(\mathrm{mL}) \times$ average radiologic lung density $(\mathrm{g} / \mathrm{mL})$ 


\section{Histologic analysis}

After six months of CT scanning, all animals were euthanized using an appropriate amount of anesthetic pentobarbital. The euthanized animals were then placed in the supine position to perform tracheostomy. When lungs collapsed following a small intercostal incision, we continued to instill $2.5 \%$ glutaraldehyde via airway at a $25 \mathrm{~cm} \mathrm{H}_{2} \mathrm{O}$ pressure above the sternum in the thoracic cavity for one hour. After 1-hour of in vivo tissue fixing, the lung was extracted from the thoracic cavity and was subsequently immersed in glutaraldehyde for 24 hours.

The anatomical lung volume was then measured using the immersion method ${ }^{19}$, and it was defined as the average of the two saline volume displacement measurements.

Tissue blocks were sampled from five different parts from each left lung. The sampling parts were randomly selected and adopted for all lungs. The tissue blocks were then fixed again with formaldehyde and were stained with Hematoxylin and Eosin.

The histologic proportion of lung parenchyma in the lung was calculated using the ImageJ software 20,21 . In this process, we performed background subtraction to correct for uneven illuminated background, conversion to 8-bit image data and reconstruction in black and white images, and we finally measured the area of lung parenchyma (Supplemental material 2). A magnification 40x was used for evaluation. The histologic lung parenchymal amount was defined as follows:

Histologic parenchymal amount $=$ anatomical lung volume $\times \frac{\text { the area of lung parenchyma }}{\text { the whole area }}$

\section{Statistical analysis}

Statistical analyses were performed with EZR (Saitama Medical Center, Jichi Medical University), which is a graphical user interface for $\mathrm{R}$ (The R Foundation for Statistical Computing, version 2.13.0) ${ }^{22}$, and the acquired values were expressed as mean \pm standard deviation. The Shapilo-Wilk test was used for testing normal distribution. All animal characteristics were analyzed using the Kruskal-Wallis test. All data were analyzed by using one-way ANOVA, and we then used the Tukey's post-hoc test to compare the values between the three groups. Finally, the Pearson's correlation coefficient was calculated in order to detect potential correlation between the two variables. $P$-values less than 0.05 were considered statistically significant.

\section{Declarations}

\section{Author contributions}

K.O., T.Y., and H.D. contributed to the hypothesis development and the experimental design. K.O. wrote the paper. K.O., T.Y, and M.H. performed the canine experiments. All authors contributed to the data interpretation and reviewed the manuscript. 


\section{References}

1. Davies, G. \& Reid, L. Growth of the alveoli and pulmonary arteries in childhood. Thorax. 25, 669-681 (1970).

2. Thurlbeck, W. M. Postnatal human lung growth. Thorax. 37, 564-571 (1982).

3. Butler, J. P. et al. Evidence for Adult Lung Growth in Humans. N. Engl. J. Med. 367, 244-247 (2012).

4. Madani, A., Zanen, J., de Maertelaer, V. \& Gevenois, P. A. Pulmonary emphysema: objective quantification at multi-detector row CT-comparison with macroscopic and microscopic morphometry. Radiology. 238, 1036-1043 (2006).

5. Humphries, S. M. et al. Quantitative high-resolution computed tomography fibrosis score: performance characteristics in idiopathic pulmonary fibrosis. Eur. Respir. J.52, (2018).

6. Gattinoni, L., Caironi, P., Pelosi, P. \& Goodman, L. R. What has computed tomography taught us about the acute respiratory distress syndrome? Am. J. Respir. Crit. Care Med. 164, 1701-1711 (2001).

7. Ravikumar, P. et al. Regional lung growth following pneumonectomy assessed by computed tomography. J. Appl. Physiol. 97, 1567-74; discussion 1549(2004).

8. Yilmaz, C. et al. Noninvasive quantification of heterogeneous lung growth following extensive lung resection by high-resolution computed tomography. J. Appl. Physiol. 107, 1569-1578 (2009).

9. Ravikumar, P. et al. Separating in vivo mechanical stimuli for postpneumonectomy compensation: imaging and ultrastructural assessment. J. Appl. Physiol. 114, 961-970 (2013).

10. Mizobuchi, T. et al. Radiologic evaluation for volume and weight of remnant lung in living lung donors. J. Thorac. Cardiovasc. Surg. 146, 1253-1258 (2013).

11. Shikuma, K. et al. Radiologic and Functional Analysis of Compensatory Lung Growth After LivingDonor Lobectomy. Ann. Thorac. Surg. 105, 909-914 (2018).

12. Chen-Yoshikawa, T. F. \& Date, H. Update on three-dimensional image reconstruction for preoperative simulation in thoracic surgery. J. Thorac. Dis. 8, S295-301 (2016).

13. Chen-Yoshikawa, T. F. \& Date, H. Three-dimensional image in lung transplantation. Gen. Thorac. Cardiovasc. Surg. 66, 19-26 (2018).

14. Paisley, D., Bevan, L., Choy, K. J. \& Gross, C. The pneumonectomy model of compensatory lung growth: insights into lung regeneration. Pharmacol. Ther. 142, 196-205 (2014).

15. Hsia, C. C., Herazo, L. F., Fryder-Doffey, F. \& Weibel, E. R. Compensatory lung growth occurs in adult dogs after right pneumonectomy. J. Clin. Invest. 94, 405-412 (1994).

16. Hsia, C. C. et al. Structural changes underlying compensatory increase of diffusing capacity after left pneumonectomy in adult dogs. J. Clin. Invest. 92, 758-764 (1993).

17. Hsia, C. C. W., Hyde, D. M., Ochs, M. \& Weibel, E. R. How to measure lung structure-what for? On the 'Standards for the quantitative assessment of lung structure'. Respiratory physiology \& neurobiology. 171, 72-74 (2010). 
18. Yilmaz, C. et al. Progressive adaptation in regional parenchyma mechanics following extensive lung resection assessed by functional computed tomography. J. Appl. Physiol. 111, 1150-1158 (2011).

19. Scherle, W. A simple method for volumetry of organs in quantitative stereology. Mikroskopie. 26, 5760 (1970).

20. Schneider, C. A., Rasband, W. S. \& Eliceiri, K. W. NIH Image to Image J: 25 years of image analysis. Nat. Methods. 9, 671-675 (2012).

21. Chung, S. H. \& Bae, C. W. Association of Surfactant Protein with Expression of Hoxa5 and Hoxb5 in Rabbit Fetal Lung. Int. J. Med. Sci. 14, 1189-1196 (2017).

22. Kanda, Y. Investigation of the freely available easy-to-use software 'EZR' for medical statistics. Bone Marrow Transplant. 48, 452-458 (2013).

\section{Figures}

a

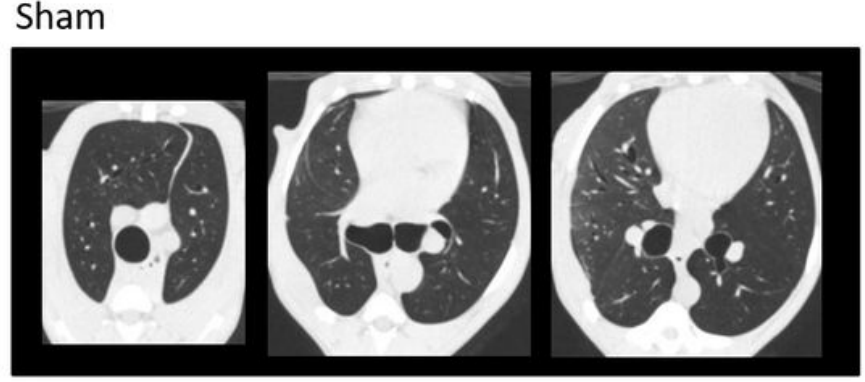

Bilobectomy

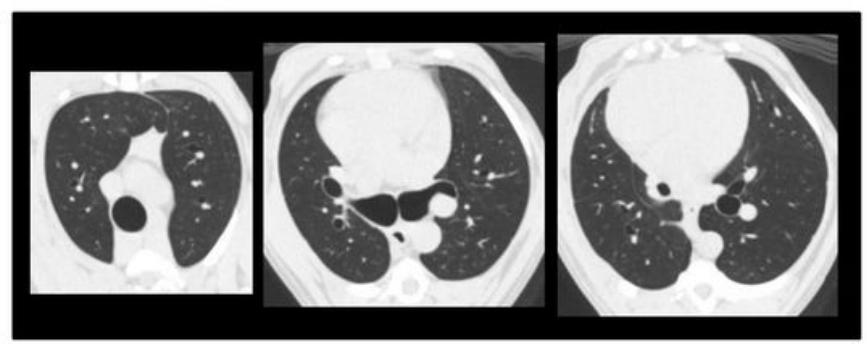

Pneumonectomy

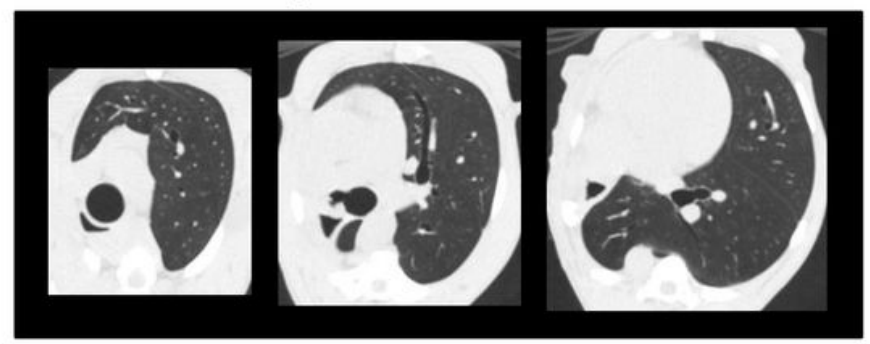

b

Radiologic lung volume (ml)

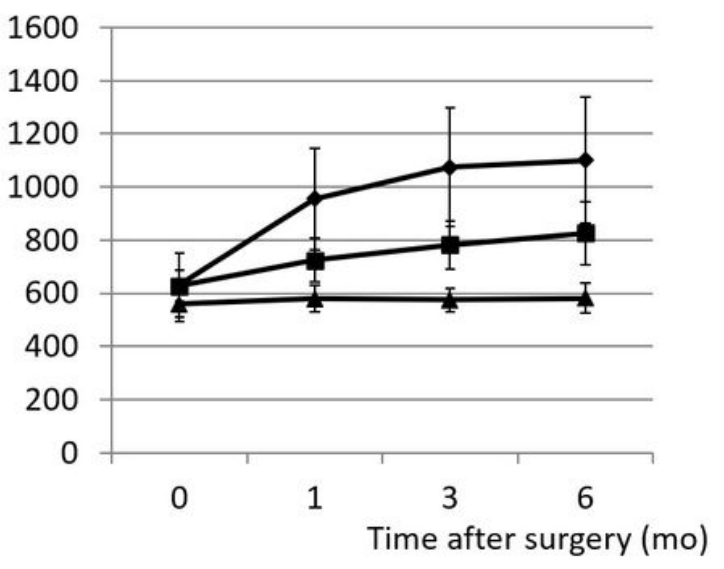

- Pneumonectomy

- Bilobectomy

- Sham

\section{Figure 1}

a: Representative computed tomography (CT) scan images 6 months after surgery of the Pneumonectomy, Bilobectomy, and Sham groups. b) The time course of lung volume measured from CT 
data.

a

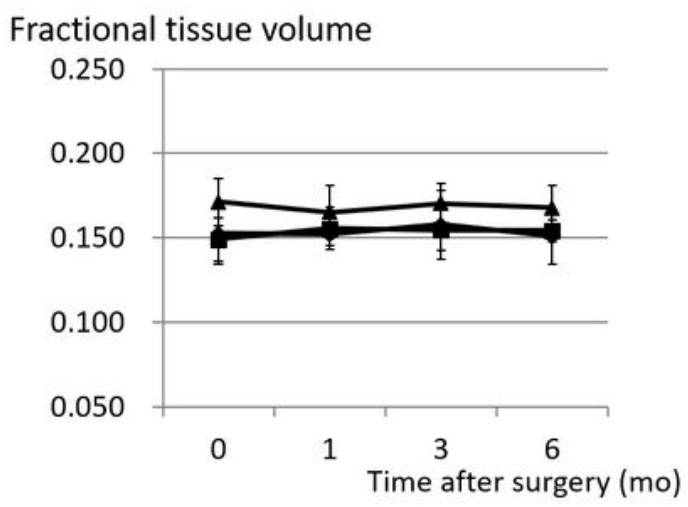

c

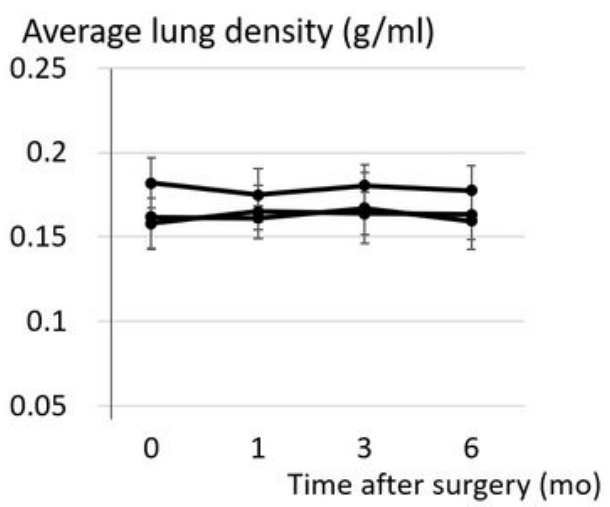

b

Radiologic tissue volume (ml)

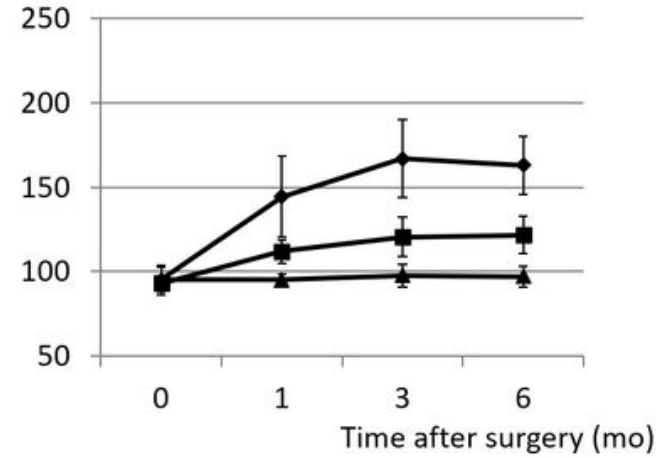

d

Radiologic lung weight (g)

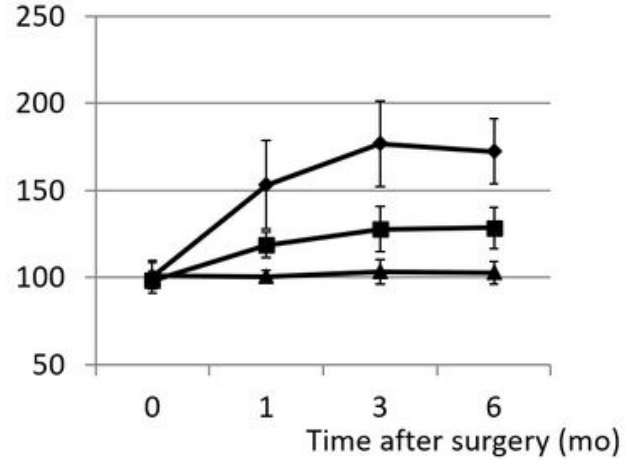

e

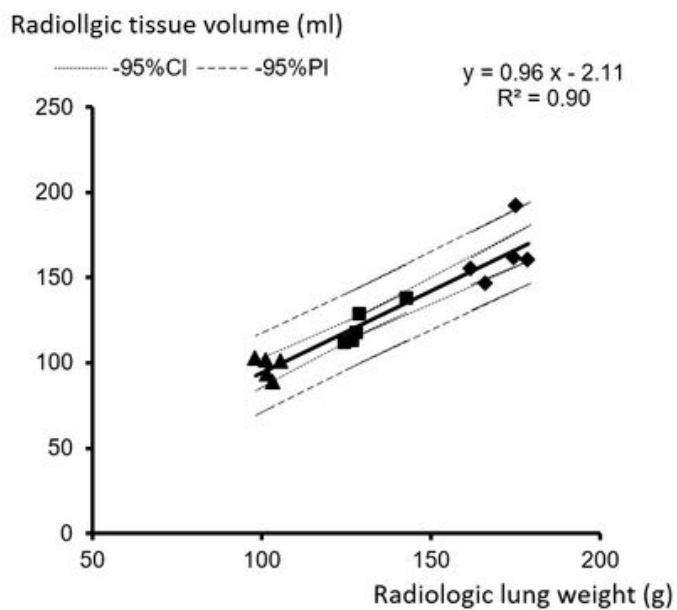

Pneumonectomy

- Bilobectomy

A Sham

\section{Figure 2}

$\mathrm{a}, \mathrm{b}$ : The value changes of the fractional tissue volume (a) and of the radiologic tissue volume (b). c, d: The value changes of the average lung density (c) and of the radiologic lung weight (d). e: Correlation between radiologic tissue volume and radiologic lung weight. 
a

Anatomic lung volume of fixed whole left lung (ml)

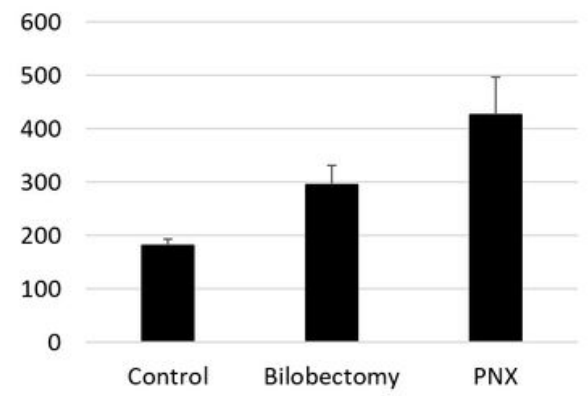

b

Sham

Bilobectomy

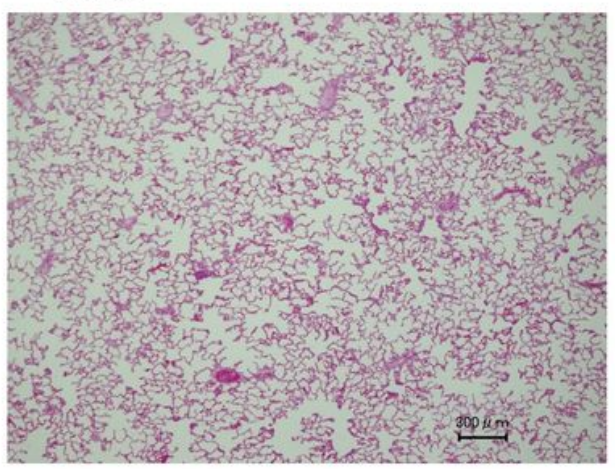

C

Proportion of lung parenchyma

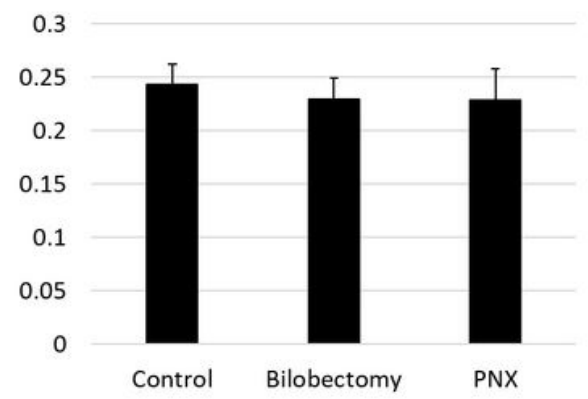

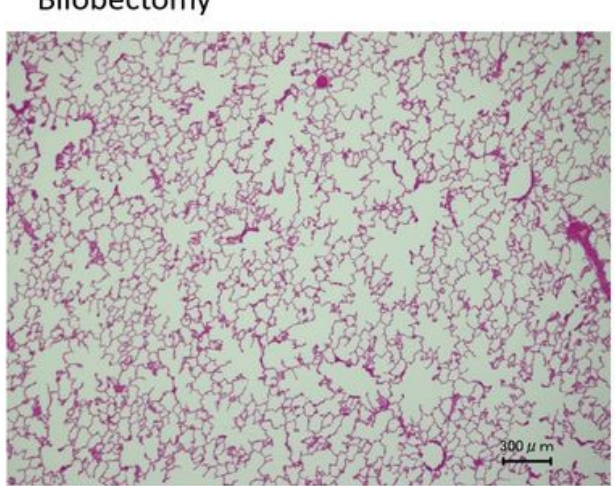

d

Histologic parenchymal amount (ml)

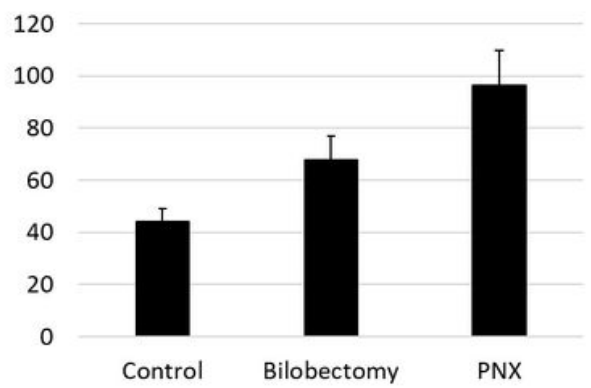

Pneumonectomy

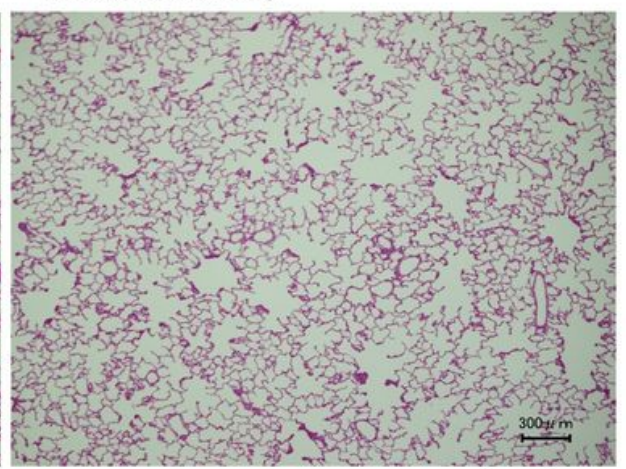

$(\times 4)$

\section{Figure 3}

a: The bar graph shows anatomical lung volume of the fixed left lungs six months after surgery of the Sham, Bilobectomy, and Pneumonectomy groups. b: The representative histologic findings of residual left lungs of the Sham, Bilobectomy, and Pneumonectomy groups. Scale bar: $300 \mu \mathrm{m}$. c, d: The bar graphs of the proportions of lung parenchyma (c) and the histologic parenchymal amounts (d) of the Sham, Bilobectomy, and Pneumonectomy groups. PNX: pneumonectomy. 
a Radiolgic tissue volume ( $\mathrm{ml}$ )

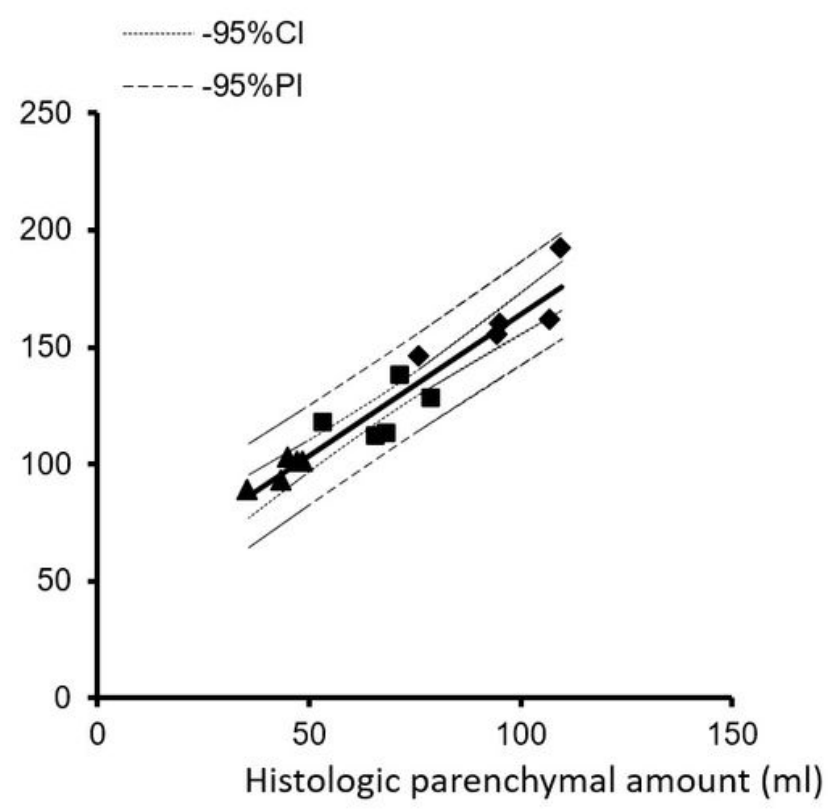

b Radiologic lung weight (g)

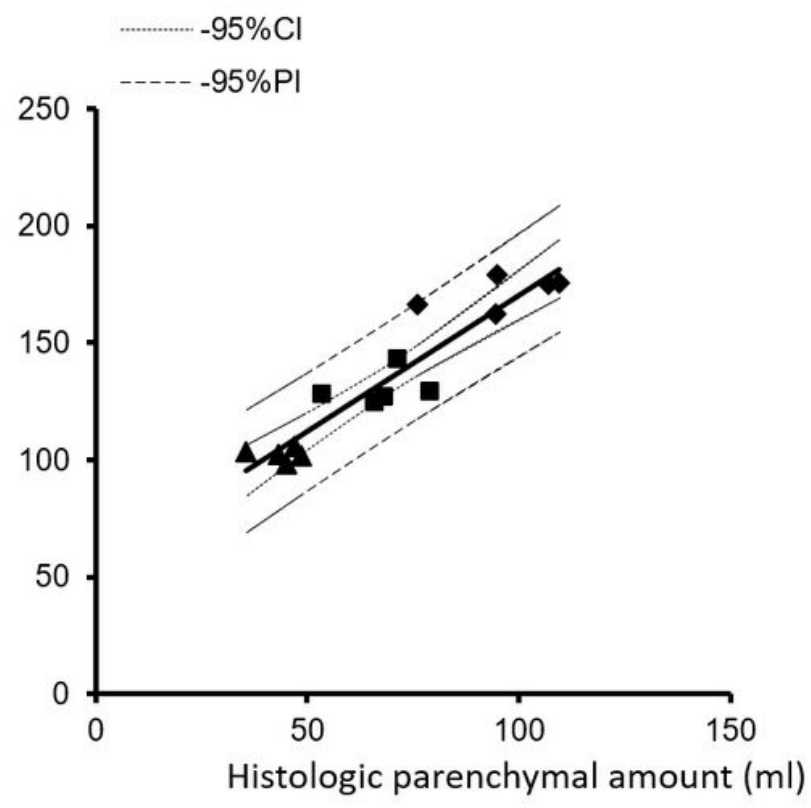

Pneumonectomy

Bilobectomy

$\Delta$ Sham

\section{Figure 4}

Correlation of the values of lung parenchymal amount measured by computed tomography analysis (a: radiologic tissue volume, b: radiologic lung weight) and by histologic morphometry. 
Surgery $\quad$ C T

Pneumonectomy $(n=5)$

Preoperative

scanning

Bilobectomy $(n=5)$

Control thoracotomy $(n=5)$

\section{1 month}

3 months

6 months

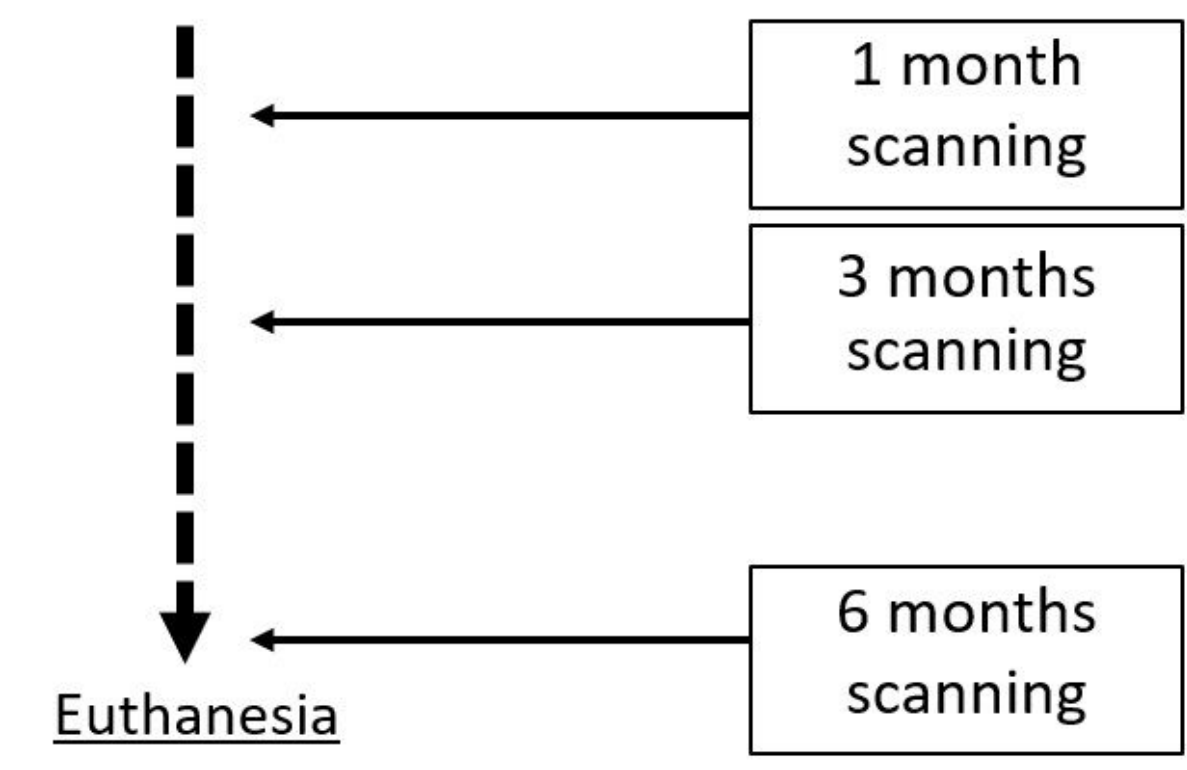

Lung fixation

Figure 5

A diagram of the experimental protocol. Chest computed tomography (CT) scans to all animals preceded surgical procedures in order to establish a baseline. We performed control right thoracotomy, right lower and cardiac lobes, or right pneumonectomy to the animals included in this study ( $n=5$ in each group). CT scans were also taken at the postoperative time-points of 1, 3, and 6 months after surgery. Following six months of postoperative scanning, the animals were euthanized, and their lung-heart blocks were retrieved.

\section{Supplementary Files}

This is a list of supplementary files associated with this preprint. Click to download.

- Supplimentalfile1.tif 
- Supplimentalfile2.tif

Page 16/16 